\title{
FINITE ELEMENT METHODS FOR THE DISPLACEMENT OBSTACLE PROBLEM OF CLAMPED PLATES
}

\author{
SUSANNE C. BRENNER, LI-YENG SUNG, AND YI ZHANG
}

\begin{abstract}
We study finite element methods for the displacement obstacle problem of clamped Kirchhoff plates. A unified convergence analysis is provided for $C^{1}$ finite element methods, classical nonconforming finite element methods and $C^{0}$ interior penalty methods. Under the condition that the obstacles are sufficiently smooth and that they are separated from each other and the zero displacement boundary constraint, we prove that the convergence in the energy norm is $O(h)$ for convex domains.
\end{abstract}

\section{INTRODUCTION}

Let $\Omega \subset \mathbb{R}^{2}$ be a bounded convex polygonal domain, $f \in L_{2}(\Omega), \psi_{1}, \psi_{2} \in$ $C^{2}(\Omega) \cap C(\bar{\Omega}), \psi_{1}<\psi_{2}$ on $\bar{\Omega}$, and $\psi_{1}<0<\psi_{2}$ on $\partial \Omega$. In this paper we consider the following displacement obstacle problem for a clamped Kirchhoff plate:

Find $u \in K$ such that

$$
u=\underset{v \in K}{\operatorname{argmin}} G(v)
$$

where

$$
\begin{aligned}
K & =\left\{v \in H_{0}^{2}(\Omega): \psi_{1} \leq v \leq \psi_{2} \quad \text { on } \quad \Omega\right\}, \\
G(v) & =\frac{1}{2} a(v, v)-(f, v), \\
a(w, v) & =\int_{\Omega} D^{2} w: D^{2} v d x \text { and }(f, v)=\int_{\Omega} f v d x .
\end{aligned}
$$

Here $D^{2} w: D^{2} v=\sum_{i, j=1}^{2} w_{x_{i} x_{j}} v_{x_{i} x_{j}}$ is the Frobenius inner product between the Hessian matrices of $w$ and $v$.

Since $a(\cdot, \cdot)$ is symmetric, bounded and coercive on $H_{0}^{2}(\Omega)$ and $K$ is a nonempty closed convex subset of $H_{0}^{2}(\Omega)$, by the standard theory (cf. [41, 38, 45, 32]) the obstacle problem (1.1) has a unique solution that is also uniquely determined by the variational inequality

$$
a(u, v-u) \geq(f, v-u) \quad \forall v \in K .
$$

Received by the editor November 13, 2010 and, in revised form, November 14, 2010 and May 4, 2011.

2010 Mathematics Subject Classification. Primary 65K15, 65N30.

Key words and phrases. Displacement obstacle, clamped Kirchhoff plate, fourth order, variational inequality, finite element, discontinuous Galerkin.

This work was supported in part by the National Science Foundation under Grant No. DMS10-16332 and by the Institute for Mathematics and its applications with funds provided by the National Science Foundation. 
Remark 1.1. One can also use an equivalent formulation of (1.1) where the bilinear form $a(\cdot, \cdot)$ is given by $a(w, v)=\int_{\Omega}(\Delta w)(\Delta v) d x$. However, the choice of $a(\cdot, \cdot)$ in (1.4) is more appropriate for nonconforming finite element methods because the corresponding norms provide more local information.

Finite element methods for second order displacement obstacle problems have been investigated in [29, 20, 24, 34, 49, 36, 51]. An important ingredient in their $a$ priori error analysis is the $H^{2}$ (and higher) regularity of the solution of the obstacle problem which holds if the domain and the data are sufficiently smooth. The main difference/difficulty in the a priori error analysis of finite element methods for the fourth order obstacle problem (1.1) is the lack of $H^{4}$ regularity for the solution $u$.

It is known (cf. [30, 31, 21, 46, 32]) that $u \in H_{l o c}^{3}(\Omega) \cap C^{2}(\Omega)$ under the assumptions on $\psi_{i}$. Since $\psi_{i} \in C(\bar{\Omega})$ and $\psi_{1}<0<\psi_{2}$ on $\partial \Omega$, we have $\psi_{1}<u<\psi_{2}$ in a neighborhood $\mathcal{N}$ of $\partial \Omega$. Then (1.5) implies $\Delta^{2} u=f$ in $\mathcal{N}$ and hence $u \in H^{3}(\mathcal{N})$ by the convexity of $\Omega$ (cf. [8, 35, 26, 39]). It follows that $u \in H^{3}(\Omega)$. However, in general $u$ does not belong to $H_{l o c}^{4}(\Omega)$ even when $f, \psi_{i}$ and $\partial \Omega$ are smooth (cf. [21]). As far as we know, a thorough analysis of finite element methods for (1.1) is still missing from the literature because of the lack of $H^{4}$ regularity.

Remark 1.2. The $C^{2}$ regularity result in [31, 21] is obtained for the one-obstacle problem and $f=0$. But it can be extended in a standard fashion to the twoobstacle problem (1.1) under our assumptions on $\psi_{1}, \psi_{2}$ and $f$ (cf. Appendix A). Note also that $u$ does not belong to $C^{2}(\Omega)$ in general if we only assume $\psi_{1} \leq \psi_{2}$ in $\Omega$ (cf. [22]).

Remark 1.3. Mixed finite element methods for fourth order obstacle problems were discussed in 34] without convergence rates. Nonconforming finite element methods were studied in [50], where an $O(h)$ error estimate was obtained for convex domains. However, in that paper $\Delta^{2} u$ is erroneously treated as a function in $L_{2}(\Omega)$. Discontinuous Galerkin methods were also investigated in [4] under a mistaken $H^{4}$ regularity of $u$.

In this paper we take advantage of the fact that $H^{2}(\Omega)$ is compactly embedded in $C(\bar{\Omega})$ to give a convergence analysis that uses only the minimization/variational inequality formulations of the continuous and discrete problems. We prove that the error in the energy norm is of magnitude $O(h)$.

The rest of the paper is organized as follows. We introduce three types of finite element methods in Section 2 and present a general framework for the analysis of these methods. In Section 3 we study an auxiliary obstacle problem that plays a key role in the convergence analysis of the finite element methods, which is carried out in Section 4. We end the paper with some concluding remarks in Section [5]and three appendices. The extension of the $C^{2}$ regularity result to the two-obstacle problem is discussed in Appendix A. Appendix B contains an estimate for the Morley element and Appendix $\mathrm{C}$ provides a concise unified analysis of finite element methods for the biharmonic problem using the tools introduced in Section 2. 


\section{A GENERAL FRAMEWORK FOR FINITE ELEMENT METHODS FOR THE OBSTACLE PROBLEM}

Let $\mathcal{T}_{h}$ be a regular triangulation of $\Omega$ with mesh size $h$. The piecewise Sobolev space $H^{3}\left(\Omega, \mathcal{T}_{h}\right)$ is defined by

$$
H^{3}\left(\Omega, \mathcal{T}_{h}\right)=\left\{v \in L_{2}(\Omega): v_{T}=\left.v\right|_{T} \in H^{3}(T) \quad \forall T \in \mathcal{T}_{h}\right\} .
$$

Remark 2.1. The energy space $H^{3}\left(\Omega, \mathcal{T}_{h}\right)$ is needed for $C^{0}$ interior penalty methods (cf. Example 2.4). We can use the larger space $H^{2}\left(\Omega, \mathcal{T}_{h}\right)=\left\{v \in L_{2}(\Omega): v_{T}=\right.$ $\left.\left.v\right|_{T} \in H^{2}(T) \quad \forall T \in \mathcal{T}_{h}\right\}$ for the classical nonconforming finite element methods (cf. Example 2.3).

Let $V_{h}$ be a finite element space associated with $\mathcal{T}_{h}$ and $a_{h}(\cdot, \cdot)$ be a symmetric bilinear form on $H^{3}\left(\Omega, \mathcal{T}_{h}\right)$ such that

$$
a_{h}(w, v)=a(w, v) \quad \forall v, w \in H_{0}^{2}(\Omega) \cap H^{3}\left(\Omega, \mathcal{T}_{h}\right) .
$$

We assume there exists a norm $\|\cdot\|_{h}$ on $V_{h}+\left(H_{0}^{2}(\Omega) \cap H^{3}\left(\Omega, \mathcal{T}_{h}\right)\right)$ such that

$$
\begin{aligned}
\left|a_{h}(v, w)\right| \leq C_{1}\|v\|_{h}\|w\|_{h} & & \forall v, w \in V_{h}+\left(H_{0}^{2}(\Omega) \cap H^{3}\left(\Omega, \mathcal{T}_{h}\right)\right), \\
a_{h}(v, v) \geq C_{2}\|v\|_{h}^{2} & & \forall v \in V_{h} .
\end{aligned}
$$

From here on we use $C$ (with or without subscripts) to denote a generic positive constant independent of $h$ that can take different values at different appearances.

Let $\mathcal{V}_{h}$ be the set of the vertices of $\mathcal{T}_{h}$. We assume the functions in $V_{h}$ are continuous at the vertices $p \in \mathcal{V}_{h}$ and there exists an operator $\Pi_{h}: H_{0}^{2}(\Omega) \longrightarrow V_{h}$ such that

$$
\begin{array}{cl}
\left(\Pi_{h} \zeta\right)(p)=\zeta(p) & \forall \zeta \in H_{0}^{2}(\Omega), p \in \mathcal{V}_{h}, \\
\left\|\zeta-\Pi_{h} \zeta\right\|_{h} \leq C h|\zeta|_{H^{3}(\Omega)} & \forall \zeta \in H^{3}(\Omega) \cap H_{0}^{2}(\Omega) .
\end{array}
$$

Furthermore, we assume there exists an operator $E_{h}: V_{h} \longrightarrow H_{0}^{2}(\Omega) \cap H^{3}\left(\Omega, \mathcal{T}_{h}\right)$ such that, for any $v \in V_{h}$ and $\zeta \in H^{3}(\Omega) \cap H_{0}^{2}(\Omega)$,

$$
\begin{aligned}
& \left(E_{h} v\right)(p)=v(p) \quad \forall p \in \mathcal{V}_{h} \\
& \left\|v-E_{h} v\right\|_{L_{2}(\Omega)}+h\left(\sum_{T \in \mathcal{T}_{h}}\left|v-E_{h} v\right|_{H^{1}(T)}^{2}\right)^{\frac{1}{2}}+h^{2}\left|E_{h} v\right|_{H^{2}(\Omega)} \leq C h^{2}\|v\|_{h} \\
& \left|a_{h}\left(\zeta, v-E_{h} v\right)\right| \leq C h|\zeta|_{H^{3}(\Omega)}\|v\|_{h} \\
& \left\|\zeta-E_{h} \Pi_{h} \zeta\right\|_{L_{2}(\Omega)}+h\left|\zeta-E_{h} \Pi_{h} \zeta\right|_{H^{1}(\Omega)}+h^{2}\left|\zeta-E_{h} \Pi_{h} \zeta\right|_{H^{2}(\Omega)} \\
& \quad \leq C h^{3}|\zeta|_{H^{3}(\Omega)}
\end{aligned}
$$

Example 2.2 $\left(C^{1}\right.$ Finite Elements [9, [5, 52, 23]). We take $V_{h} \subset H_{0}^{2}(\Omega)$ to be the Hsieh-Clough-Tocher macro element space or the quintic Argyris finite element space, $a_{h}(\cdot, \cdot)=a(\cdot, \cdot),\|\cdot\|_{h}=\sqrt{a(\cdot, \cdot)}=|\cdot|_{H^{2}(\Omega)}, \Pi_{h}$ to be a quasi-local interpolation operator [25, 47, 33, and $E_{h}$ to be the natural injection. The properties (2.6) - 2.9) are trivial in this case. In the case where $\Omega$ can be triangulated by rectangles, we can also use the Bogner-Fox-Schmit element. 
Example 2.3 (Classical Nonconforming Finite Elements [2, 7, 43, 27, 48]). Let $V_{h} \subset L_{2}(\Omega)$ be the Morley finite element. We take

$$
a_{h}(w, v)=\sum_{T \in \mathcal{T}_{h}} \int_{T} D^{2} w: D^{2} v d x
$$

$\|\cdot\|_{h}=\sqrt{a_{h}(\cdot, \cdot)}, \Pi_{h}$ to be the interpolation operator determined by the function values at the vertices and the integrals of the normal derivatives on the edges, and $E_{h}$ to be an enriching operator defined by averaging. Details about the construction of $E_{h}$ can be found in [13, 14, where the properties (2.6), (2.7) and (2.9) are established. The derivation of estimate (2.8) is given in Appendix B. Similar constructions (cf. [12]) can also be carried out for the Zienkiewicz element and the de Veubeke element, and in the case where $\Omega$ can be triangulated by rectangles, also for the Adini element and the incomplete biquadratic element.

Example $2.4\left(C^{0}\right.$ Interior Penalty Methods [28, 17]). These are discontinuous Galerkin methods. We take $V_{h} \subset H_{0}^{1}(\Omega)$ to be a $P_{k}$ Lagrange finite element space $(k \geq 2)$ and

$$
\begin{aligned}
& a_{h}(w, v)=\sum_{T \in \mathcal{T}_{h}} \int_{T} D^{2} w: D^{2} v d x+\sum_{e \in \mathcal{E}_{h}} \int_{e}\left\{\left\{\partial^{2} w / \partial n^{2}\right\}\right\}[[\partial v / \partial n]] d s \\
& \left.\quad+\sum_{e \in \mathcal{E}_{h}} \int_{e}\left\{\left\{\partial^{2} v / \partial n^{2}\right\}\right\}[\partial \partial / \partial n]\right] d s+\sigma \sum_{e \in \mathcal{E}_{h}}|e|^{-1} \int_{e}[[\partial w / \partial n]][\partial v / \partial n] d s,
\end{aligned}
$$

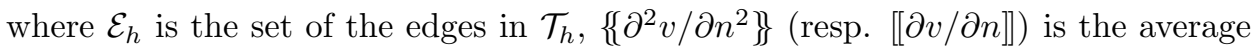
(resp. jump) of the second (resp. first) order normal derivative of $v$ across an edge, $|e|$ is the length of the edge $e$ and $\sigma>0$ is a penalty parameter. The norm $\|\cdot\|_{h}$ is defined by

$$
\|v\|_{h}^{2}=\sum_{T \in \mathcal{T}_{h}}|v|_{H^{2}(T)}^{2}+\sum_{e \in \mathcal{E}_{h}}|e|\left\|\left\{\left\{\partial^{2} v / \partial n^{2}\right\}\right\}\right\|_{L_{2}(e)}^{2}+\sum_{e \in \mathcal{E}_{h}}|e|^{-1}\|[[\partial v / \partial n]]\|_{L_{2}(e)}^{2} .
$$

The penalty parameter $\sigma$ is assumed to be large enough so that (2.3) holds. We take $\Pi_{h}$ to be the nodal interpolation operator and $E_{h}$ to be an enriching operator defined by averaging. Details of the construction of $E_{h}$ can be found in [17, where the properties (2.6), (2.7) and (2.9) are established. Property (2.8) is a simple consequence of integration by parts and (2.7).

Remark 2.5. The estimates (2.7) and (2.8) are useful tools for analyzing finite element methods for the biharmonic problem (cf. Appendix C). The estimates (2.7) and (2.9) are also useful for the analysis of fast solvers for the resulting discrete problems [11, 13, 14, 15].

The finite element method for (1.1) (equivalently (1.5)) is: Find $u_{h} \in K_{h}$ such that

$$
u_{h}=\underset{v \in K_{h}}{\operatorname{argmin}} G_{h}(v),
$$

where

$$
K_{h}=\left\{v \in V_{h}: \psi_{1}(p) \leq v(p) \leq \psi_{2}(p) \quad \forall p \in \mathcal{V}_{h}\right\},
$$

and $G_{h}(v)=\frac{1}{2} a_{h}(v, v)-(f, v)$. 
Since $a_{h}(\cdot, \cdot)$ is symmetric positive definite on $V_{h}$, it follows from the standard theory that the discrete obstacle problem (2.10) has a unique solution $u_{h}$, which is also characterized by the following variational inequality:

$$
a_{h}\left(u_{h}, v-u_{h}\right) \geq\left(f, v-u_{h}\right) \quad \forall v \in K_{h} .
$$

The finite element function $u_{h}$ provides an approximation to $u$. A preliminary error estimate is given in the following lemma.

Lemma 2.6. There exist positive constants $C_{\dagger}$ and $C_{\ddagger}$ independent of $h$ such that

$$
\left\|u-u_{h}\right\|_{h}^{2} \leq C_{\dagger}\left\|\Pi_{h} u-u\right\|_{h}^{2}+C_{\ddagger}\left[a_{h}\left(u, \Pi_{h} u-u_{h}\right)-\left(f, \Pi_{h} u-u_{h}\right)\right] .
$$

Proof. From (2.2), (2.3), (2.12) and the arithmetic-geometric mean inequality, we have

$$
\begin{aligned}
& \left\|\Pi_{h} u-u_{h}\right\|_{h}^{2} \leq C_{1} a_{h}\left(\Pi_{h} u-u_{h}, \Pi_{h} u-u_{h}\right) \\
& \quad=C_{1}\left[a_{h}\left(\Pi_{h} u-u, \Pi_{h} u-u_{h}\right)+a_{h}\left(u, \Pi_{h} u-u_{h}\right)-a_{h}\left(u_{h}, \Pi_{h} u-u_{h}\right)\right] \\
& \quad \leq C_{2}\left\|\Pi_{h} u-u\right\|_{h}\left\|\Pi_{h} u-u_{h}\right\|_{h}+C_{1}\left[a_{h}\left(u, \Pi_{h} u-u_{h}\right)-\left(f, \Pi_{h} u-u_{h}\right)\right] \\
& \quad \leq \frac{1}{2}\left\|\Pi_{h} u-u_{h}\right\|_{h}^{2}+C_{3}\left\|\Pi_{h} u-u\right\|_{h}^{2}+C_{1}\left[a_{h}\left(u, \Pi_{h} u-u_{h}\right)-\left(f, \Pi_{h} u-u_{h}\right)\right],
\end{aligned}
$$

and hence

$$
\left\|\Pi_{h} u-u_{h}\right\|_{h}^{2} \leq 2 C_{3}\left\|\Pi_{h} u-u\right\|_{h}^{2}+2 C_{1}\left[a_{h}\left(u, \Pi_{h} u-u_{h}\right)-\left(f, \Pi_{h} u-u_{h}\right)\right] .
$$

The estimate (2.13) then follows from the triangle inequality.

In view of (2.5), it only remains to find an estimate for the term

$$
a_{h}\left(u, \Pi_{h} u-u_{h}\right)-\left(f, \Pi_{h} u-u_{h}\right)
$$

on the right-hand side of (2.13) .

\section{An AUXiliary obstaCle PROBLEM}

The analysis of the term $a_{h}\left(u, \Pi_{h} u-u_{h}\right)-\left(f, \Pi_{h} u-u_{h}\right)$ should of course involve the variational inequality (1.5). But it is not easy to connect $K_{h}$ directly to $K$. However, by (1.2), (2.6) and (2.11), we have

$$
E_{h} v \in \tilde{K}_{h} \quad \forall v \in K_{h},
$$

where $\tilde{K}_{h} \subset H_{0}^{2}(\Omega)$ is defined by

$$
\tilde{K}_{h}=\left\{v \in H_{0}^{2}(\Omega): \psi_{1}(p) \leq v(p) \leq \psi_{2}(p) \quad \forall p \in \mathcal{V}_{h}\right\}
$$

Thus we are led to consider the following intermediate obstacle problem: Find $\tilde{u}_{h} \in \tilde{K}_{h}$ such that

$$
\tilde{u}_{h}=\underset{v \in \tilde{K}_{h}}{\operatorname{argmin}} G(v),
$$

where the functional $G$ is given by (1.3)-(1.4).

The unique solution of (3.2) satisfies the following variational inequality:

$$
a\left(\tilde{u}_{h}, v-\tilde{u}_{h}\right) \geq\left(f, v-\tilde{u}_{h}\right) \quad \forall v \in \tilde{K}_{h} .
$$

Our strategy is to first estimate the difference between $u$ and $\tilde{u}_{h}$ in this section and then combine it with the variational inequalities (1.5) and (3.3) to complete the convergence analysis in Section 4 . 
Note that $K$ is a subset of $\tilde{K}_{h}$ and the problems (1.1) and (3.2) involve the same functional $G$. We can therefore consider $u$ as an internal approximation of $\tilde{u}_{h}$ and it is known (cf. [6] and Remark 3.5) that the distance between $\tilde{u}_{h}$ and $u$ can be bounded by the square root of the distance between $\tilde{u}_{h}$ and $K$. Below we will estimate the distance between $\tilde{u}_{h}$ and $K$ through several lemmas.

Lemma 3.1. There exists a positive constant $C$ independent of $h$ such that

$$
\left\|\tilde{u}_{h}\right\|_{H^{2}(\Omega)} \leq C .
$$

Proof. Since $K \subset \tilde{K}_{h}$, we deduce from (3.2) that

$$
G\left(\tilde{u}_{h}\right) \leq G(u)
$$

and hence, by the Cauchy-Schwarz inequality, a Poincaré-Friedrichs inequality [44] and the arithmetic-geometric mean inequality,

$$
\begin{aligned}
\frac{1}{2}\left|\tilde{u}_{h}\right|_{H^{2}(\Omega)}^{2} & \leq G(u)+\int_{\Omega} f \tilde{u}_{h} d x \\
& \leq G(u)+\|f\|_{L_{2}(\Omega)}\left\|\tilde{u}_{h}\right\|_{L_{2}(\Omega)} \leq G(u)+C\|f\|_{L_{2}(\Omega)}^{2}+\frac{1}{4}\left|\tilde{u}_{h}\right|_{H^{2}(\Omega)}^{2} .
\end{aligned}
$$

Lemma 3.2. The solution $\tilde{u}_{h}$ of (3.2) converges uniformly on $\Omega$ to the solution $u$ of (1.1) as $h \downarrow 0$.

Proof. It suffices to show that given any sequence $h_{n} \downarrow 0$ there exists a subsequence $h_{n_{k}}$ such that $\tilde{u}_{h_{n_{k}}}$ converges uniformly on $\Omega$ to $u$ as $k \rightarrow \infty$.

By Lemma 3.1 and the weak sequential compactness of bounded subsets of a Hilbert space [40], there exists a subsequence $h_{n_{k}}$ such that

$$
\tilde{u}_{h_{n_{k}}} \text { converges weakly to some } u_{*} \in H_{0}^{2}(\Omega) \text { as } k \rightarrow \infty \text {. }
$$

Since $H^{2}(\Omega)$ is compactly embedded in $C(\bar{\Omega})$ by the Rellich-Kondrachov Theorem [1], the weak convergence of $\tilde{u}_{h_{n_{k}}}$ implies that

$$
\tilde{u}_{h_{n_{k}}} \text { converges uniformly on } \Omega \text { to } u_{*} \text { as } k \rightarrow \infty \text {. }
$$

In view of (3.1) and the fact that the set $\bigcup_{j \geq k} \mathcal{V}_{h_{n_{j}}}$ is dense in $\Omega$ for any $k$, we obtain by (3.6) the relation $\psi_{1} \leq u_{*} \leq \psi_{2}$ on $\Omega$, i.e., $u_{*} \in K$.

Since the functional $G$ is convex and continuous, it is also weakly lower semicontinuous (cf. [19). Therefore we have, by (3.4) and (3.5),

$$
G\left(u_{*}\right) \leq \liminf _{k \rightarrow \infty} G\left(\tilde{u}_{h_{n_{k}}}\right) \leq G(u) .
$$

We conclude from (1.1) that $u_{*}=u$ and hence $\tilde{u}_{h_{n_{k}}}$ converges uniformly on $\Omega$ to $u$ by (3.6).

Let $I_{i}(i=1,2)$ be the coincidence set of the obstacle problem (1.1) defined by

$$
I_{i}=\left\{x \in \Omega: u(x)=\psi_{i}(x)\right\} .
$$

The boundary condition of $u$ and the assumptions on $\psi_{i}$ imply that the compact sets $\partial \Omega, I_{1}$ and $I_{2}$ are mutually disjoint.

For any positive $\tau$, let the compact set $I_{i, \tau}(i=1,2)$ be defined by

$$
I_{i, \tau}=\left\{x \in \bar{\Omega}: \operatorname{dist}\left(x, I_{i}\right) \leq \tau\right\} .
$$


We can choose $\tau_{i}>0(i=1,2)$ small enough so that the compact sets $I_{1,2 \tau_{1}}, I_{2,2 \tau_{2}}$ and $\partial \Omega$ remain mutually disjoint.

Lemma 3.3. There exist positive numbers $h_{0}, \gamma_{1}$ and $\gamma_{2}$ such that

$$
\begin{array}{ll}
\tilde{u}_{h}(x)-\psi_{1}(x) \geq \gamma_{1} & \text { if } x \in \bar{\Omega} \text { and } \operatorname{dist}\left(x, I_{1}\right) \geq \tau_{1}, \\
\psi_{2}(x)-\tilde{u}_{h}(x) \geq \gamma_{2} & \text { if } x \in \bar{\Omega} \text { and } \operatorname{dist}\left(x, I_{2}\right) \geq \tau_{2},
\end{array}
$$

provided $h \leq h_{0}$.

Proof. Since $u-\psi_{1}$ (resp. $\psi_{2}-u$ ) is strictly positive on the compact set $\{x \in$ $\left.\bar{\Omega}: \operatorname{dist}\left(x, I_{1}\right) \geq \tau_{1}\right\}$ (resp. $\left.\left\{x \in \bar{\Omega}: \operatorname{dist}\left(x, I_{2}\right) \geq \tau_{2}\right\}\right)$, this is an immediate consequence of Lemma 3.2

Let $\mathcal{I}_{h}$ be the nodal interpolation operator for the conforming $P_{1}$ finite element space associated with $\mathcal{T}_{h}$. We can rewrite the displacement constraints on $\tilde{u}_{h}$ as

$$
\mathcal{I}_{h} \psi_{1} \leq \mathcal{I}_{h} \tilde{u}_{h} \leq \mathcal{I}_{h} \psi_{2} \quad \text { on } \bar{\Omega} .
$$

Let the number $\delta_{h, i}$ be defined by

$$
\delta_{h, i}=\left\|\left(\tilde{u}_{h}-\mathcal{I}_{h} \tilde{u}_{h}\right)+\left(\mathcal{I}_{h} \psi_{i}-\psi_{i}\right)\right\|_{L_{\infty}\left(I_{i, \tau_{i}}\right)} \quad \text { for } \quad i=1,2 .
$$

Since $\psi_{i} \in C^{2}(\Omega)$ and the compact set $I_{i, \tau_{i}}$ is disjoint from $\partial \Omega$, Taylor's Theorem implies that

$$
\left\|\psi_{i}-\mathcal{I}_{h} \psi_{i}\right\|_{L_{\infty}\left(I_{i, \tau_{i}}\right)} \leq C h^{2} \quad \text { for } \quad i=1,2 .
$$

Moreover, from the standard interpolation error estimate (cf. [24, 16])

$$
\left\|\zeta-\mathcal{I}_{h} \zeta\right\|_{L_{\infty}(\Omega)} \leq C h|\zeta|_{H^{2}(\Omega)} \quad \forall \zeta \in H^{2}(\Omega)
$$

and Lemma 3.1, we have

$$
\left\|\tilde{u}_{h}-\mathcal{I}_{h} \tilde{u}_{h}\right\|_{L_{\infty}(\Omega)} \leq C h\left|\tilde{u}_{h}\right|_{H^{2}(\Omega)} \leq C h,
$$

and hence

$$
\delta_{h, i} \leq C h \quad \text { for } \quad i=1,2 .
$$

Lemma 3.4. There exists a positive constant $C$ independent of $h$ such that

$$
\left|u-\tilde{u}_{h}\right|_{H^{2}(\Omega)}^{2} \leq C\left(\delta_{h, 1}+\delta_{h, 2}\right)
$$

provided $h$ is sufficiently small.

Proof. Let $h_{0}, \gamma_{1}$ and $\gamma_{2}$ be as in Lemma 3.3 .

Without loss of generality, we may assume $h \leq h_{0}$. We have, by (3.11) and (3.12),

$$
\begin{aligned}
& \tilde{u}_{h}(x)-\psi_{1}(x) \\
& =\tilde{u}_{h}(x)-\mathcal{I}_{h} \tilde{u}_{h}(x)+\mathcal{I}_{h} \tilde{u}_{h}(x)-\mathcal{I}_{h} \psi_{1}(x)+\mathcal{I}_{h} \psi_{1}(x)-\psi_{1}(x) \\
& \geq-\delta_{h, 1} \quad \forall x \in I_{1, \tau_{1}}, \\
& \psi_{2}(x)-\tilde{u}_{h}(x) \\
& =\psi_{2}(x)-\mathcal{I}_{h} \psi_{2}(x)+\mathcal{I}_{h} \psi_{2}(x)-\mathcal{I}_{h} \tilde{u}_{h}(x)+\mathcal{I}_{h} \tilde{u}_{h}(x)-\tilde{u}_{h}(x) \\
& \geq-\delta_{h, 2} \quad \forall x \in I_{2, \tau_{2}} \text {. }
\end{aligned}
$$

In view of (3.15) we may also assume that, for $h \leq h_{0}$,

$$
\max _{i=1,2} \delta_{h, i}<\min _{i=1,2} \gamma_{i}
$$


Let $\phi_{i}(i=1,2)$ be a function in $C^{\infty}(\bar{\Omega})$ such that

$$
\begin{array}{cl}
0 \leq \phi_{i} \leq 1 & \text { on } \bar{\Omega}, \\
\phi_{i}=1 & \text { on } I_{i, \tau_{i},} \\
\phi_{i}=0 & \text { on } \bar{\Omega} \backslash I_{i, 2 \tau_{i}} .
\end{array}
$$

We claim that

$$
\hat{u}_{h}=\tilde{u}_{h}+\delta_{h, 1} \phi_{1}-\delta_{h, 2} \phi_{2} \in K .
$$

Since the compact sets $I_{1,2 \tau_{1}}, I_{2,2 \tau_{2}}$ and $\partial \Omega$ are mutually disjoint, it is clear from (3.22) that $\hat{u}_{h} \in H_{0}^{2}(\Omega)$. It only remains to show that

$$
\psi_{1}(x) \leq \hat{u}_{h}(x) \leq \psi_{2}(x) \quad \forall x \in \Omega,
$$

which we will establish by considering the following three possibilities: $(i) x \in$ $\Omega \backslash\left(I_{1,2 \tau_{1}} \cup I_{2,2 \tau_{2}}\right),($ ii $) x \in I_{1,2 \tau_{1}}$ and (iii) $x \in I_{2,2 \tau_{2}}$.

In view of (3.9) and (3.10), it is trivial that (3.24) holds for $x \in \Omega \backslash\left(I_{1,2 \tau_{1}} \cup I_{2,2 \tau_{2}}\right)$. For the case where $x \in I_{1,2 \tau_{1}}$, we note that (3.10), (3.19), (3.20) and (3.22) imply

$$
\hat{u}_{h}(x)=\tilde{u}_{h}(x)+\delta_{h, 1} \phi_{1}(x) \leq \psi_{2}(x)-\gamma_{2}+\delta_{h, 1}<\psi_{2}(x) .
$$

Furthermore, if $x \in I_{1,2 \tau_{1}} \backslash I_{1, \tau_{1}}$, then

$$
\hat{u}_{h}(x)=\tilde{u}_{h}(x)+\delta_{h, 1} \phi_{1}(x) \geq \psi_{1}(x)+\gamma_{1}>\psi_{1}(x)
$$

by (3.9) and (3.20). Finally, if $x \in I_{1, \tau_{1}}$, then

$$
\hat{u}_{h}(x)=\tilde{u}_{h}(x)+\delta_{h, 1} \geq \psi_{1}(x)
$$

by (3.17) and (3.21). Therefore (3.24) holds for $x \in I_{1,2 \tau_{1}}$.

The proof that (3.24) holds for $x \in I_{2,2 \tau_{2}}$ is completely analogous.

From (1.3), (1.4), and Lemma 3.1, we have

$$
\begin{aligned}
G\left(\hat{u}_{h}\right)= & \frac{1}{2} a\left(\tilde{u}_{h}+\delta_{h, 1} \phi_{1}-\delta_{h, 2} \phi_{2}, \tilde{u}_{h}+\delta_{h, 1} \phi_{1}-\delta_{h, 2} \phi_{2}\right) \\
& \quad-\left(f, \tilde{u}_{h}+\delta_{h, 1} \phi_{1}-\delta_{h, 2} \phi_{2}\right) \\
= & \frac{1}{2} a\left(\tilde{u}_{h}, \tilde{u}_{h}\right)-\left(f, \tilde{u}_{h}\right)+a\left(\tilde{u}_{h}, \delta_{h, 1} \phi_{1}-\delta_{h, 2} \phi_{2}\right) \\
& \quad+\frac{1}{2} a\left(\delta_{h, 1} \phi_{1}-\delta_{h, 2} \phi_{2}, \delta_{h, 1} \phi_{1}-\delta_{h, 2} \phi_{2}\right)-\left(f, \delta_{h, 1} \phi_{1}-\delta_{h, 2} \phi_{2}\right) \\
\leq & G\left(\tilde{u}_{h}\right)+C\left(\delta_{h, 1}+\delta_{h, 2}\right) .
\end{aligned}
$$

From (1.1), (1.3), (1.4), (3.3), (3.23) and (3.25) we then obtain

$$
\begin{aligned}
\frac{1}{2}\left|u-\tilde{u}_{h}\right|_{H^{2}(\Omega)}^{2} & =\frac{1}{2} a(u, u)-\frac{1}{2} a\left(\tilde{u}_{h}, \tilde{u}_{h}\right)-a\left(\tilde{u}_{h}, u-\tilde{u}_{h}\right) \\
& \leq \frac{1}{2} a(u, u)-\frac{1}{2} a\left(\tilde{u}_{h}, \tilde{u}_{h}\right)-\left(f, u-\tilde{u}_{h}\right) \\
& =G(u)-G\left(\tilde{u}_{h}\right) \leq G\left(\hat{u}_{h}\right)-G\left(\tilde{u}_{h}\right) \leq C\left(\delta_{h, 1}+\delta_{h, 2}\right) .
\end{aligned}
$$

Remark 3.5. According to (3.23) the distance between $\tilde{u}_{h}$ and $K$ is bounded by

$$
\left|\delta_{h, 1} \phi_{1}-\delta_{h, 2} \phi_{2}\right|_{H^{2}(\Omega)} \leq C\left(\delta_{h, 1}+\delta_{h, 2}\right),
$$

and hence the estimate (3.16) shows precisely that the distance between $\tilde{u}_{h}$ and $u$ is bounded by the square root of the distance between $\tilde{u}_{h}$ and $K$. 
We now take a closer look at the numbers $\delta_{h, 1}$ and $\delta_{h, 2}$. Since $u \in C^{2}(\Omega)$ and the compact set $I_{i, \tau_{i}}$ is disjoint from $\partial \Omega$, we have, by Taylor's Theorem,

$$
\left\|u-\mathcal{I}_{h} u\right\|_{L_{\infty}\left(I_{i, \tau_{i}}\right)} \leq C h^{2} \quad \text { for } \quad i=1,2 .
$$

Combining (3.14) and (3.26) we find

$$
\begin{aligned}
\left\|\tilde{u}_{h}-\mathcal{I}_{h} \tilde{u}_{h}\right\|_{L_{\infty}\left(I_{i, \tau_{i}}\right)} & \leq\left\|\left(u-\tilde{u}_{h}\right)-\mathcal{I}_{h}\left(u-\tilde{u}_{h}\right)\right\|_{L_{\infty}(\Omega)}+\left\|u-\mathcal{I}_{h} u\right\|_{L_{\infty}\left(I_{i, \tau_{i}}\right)} \\
& \leq C\left(h\left|u-\tilde{u}_{h}\right|_{H^{2}(\Omega)}+h^{2}\right),
\end{aligned}
$$

which together with (3.12) and (3.13) implies

$$
\delta_{h, i} \leq C\left(h\left|u-\tilde{u}_{h}\right|_{H^{2}(\Omega)}+h^{2}\right) \quad \text { for } \quad i=1,2 .
$$

It now follows from (3.16), (3.27) and the arithmetic-geometric mean inequality that

$$
\left|u-\tilde{u}_{h}\right|_{H^{2}(\Omega)}^{2} \leq C h^{2}+\frac{1}{2}\left|u-\tilde{u}_{h}\right|_{H^{2}(\Omega)}^{2},
$$

and hence we have

$$
\left|u-\tilde{u}_{h}\right|_{H^{2}(\Omega)} \leq C h .
$$

Finally, we note that (3.27) and (3.28) imply

$$
\delta_{h, i} \leq C h^{2} \quad \text { for } \quad i=1,2,
$$

which improves the estimate (3.15).

\section{Convergence Analysis}

With Lemma 3.4 the estimate (3.29), and the two variational inequalities (1.5) and (3.3) at our disposal, we can now complete the convergence analysis of the finite element methods.

In our analysis we will also need the estimate

$$
|a(\zeta, v)| \leq C|\zeta|_{H^{3}(\Omega)}|v|_{H^{1}(\Omega)} \quad \forall \zeta \in H^{3}(\Omega), v \in H_{0}^{2}(\Omega),
$$

which follows immediately from (1.4) and integration by parts.

Lemma 4.1. There exists a positive constant $C$ independent of $h$ such that

$$
\begin{aligned}
& a_{h}\left(u, \Pi_{h} u-u_{h}\right)-\left(f, \Pi_{h} u-u_{h}\right) \\
& \leq C\left[\left(h+\delta_{h, 1}^{\frac{1}{2}}+\delta_{h, 2}^{\frac{1}{2}}\right)\left\|\Pi_{h} u-u_{h}\right\|_{h}+\left(h^{2}+\delta_{h, 1}+\delta_{h, 2}\right)\right],
\end{aligned}
$$

where $\delta_{h, i}$ is defined in (3.12).

Proof. We have

$$
a_{h}\left(u, \Pi_{h} u-u_{h}\right)=a\left(u, E_{h} \Pi_{h} u-E_{h} u_{h}\right)+a_{h}\left(u, \Pi_{h} u-u_{h}-E_{h}\left(\Pi_{h} u-u_{h}\right)\right)
$$

because of (2.1), and it follows from (2.8) that

$$
\left|a_{h}\left(u, \Pi_{h} u-u_{h}-E_{h}\left(\Pi_{h} u-u_{h}\right)\right)\right| \leq C h\left\|\Pi_{h} u-u_{h}\right\|_{h} .
$$

Moreover, we have

$$
a\left(u, E_{h} \Pi_{h} u-E_{h} u_{h}\right)=a\left(\tilde{u}_{h}, E_{h} \Pi_{h} u-E_{h} u_{h}\right)+a\left(u-\tilde{u}_{h}, E_{h} \Pi_{h} u-E_{h} u_{h}\right),
$$


and it follows from (2.7) and (3.16) that

$$
\begin{aligned}
\left|a\left(u-\tilde{u}_{h}, E_{h} \Pi_{h} u-E_{h} u_{h}\right)\right| & \leq\left|u-\tilde{u}_{h}\right|_{H^{2}(\Omega)}\left|E_{h}\left(\Pi_{h} u-u_{h}\right)\right|_{H^{2}(\Omega)} \\
& \leq C\left(\delta_{h, 1}^{\frac{1}{2}}+\delta_{h, 2}^{\frac{1}{2}}\right)\left\|\Pi_{h} u-u_{h}\right\|_{h} .
\end{aligned}
$$

Now we use (3.3) to derive

$$
\begin{aligned}
a\left(\tilde{u}_{h}, E_{h} \Pi_{h} u-E_{h} u_{h}\right) & =a\left(\tilde{u}_{h}, \tilde{u}_{h}-E_{h} u_{h}\right)+a\left(\tilde{u}_{h}, E_{h} \Pi_{h} u-\tilde{u}_{h}\right) \\
& \leq\left(f, \tilde{u}_{h}-E_{h} u_{h}\right)+a\left(\tilde{u}_{h}, E_{h} \Pi_{h} u-\tilde{u}_{h}\right) .
\end{aligned}
$$

Note that

$$
\begin{gathered}
a\left(\tilde{u}_{h}, E_{h} \Pi_{h} u-\tilde{u}_{h}\right)=a\left(\tilde{u}_{h}-u, E_{h} \Pi_{h} u-\tilde{u}_{h}\right)+a\left(u, E_{h} \Pi_{h} u-u\right) \\
+a\left(u, u-\tilde{u}_{h}\right)
\end{gathered}
$$

and

$$
\begin{aligned}
& \left|a\left(\tilde{u}_{h}-u, E_{h} \Pi_{h} u-\tilde{u}_{h}\right)+a\left(u, E_{h} \Pi_{h} u-u\right)\right| \\
& \quad \leq\left|u-\tilde{u}_{h}\right|_{H^{2}(\Omega)}\left|\left(\tilde{u}_{h}-u\right)+\left(u-E_{h} \Pi_{h} u\right)\right|_{H^{2}(\Omega)} \\
& \quad+C|u|_{H^{3}(\Omega)}\left|u-E_{h} \Pi_{h} u\right|_{H^{1}(\Omega)} \\
& \quad \leq C\left(\left|u-\tilde{u}_{h}\right|_{H^{2}(\Omega)}^{2}+\left|u-E_{h} \Pi_{h} u\right|_{H^{2}(\Omega)}^{2}+|u|_{H^{3}(\Omega)}\left|u-E_{h} \Pi_{h} u\right|_{H^{1}(\Omega)}\right) \\
& \quad \leq C\left(h^{2}+\delta_{h, 1}+\delta_{h, 2}\right)
\end{aligned}
$$

by (2.9), (3.16) and (4.1).

Without loss of generality, we may assume that $h$ is sufficiently small so that (3.23) holds. Therefore we can use (1.5) to obtain

$$
\begin{aligned}
a\left(u, u-\tilde{u}_{h}\right)= & a\left(u, u-\hat{u}_{h}\right)+\delta_{h, 1} a\left(u, \phi_{1}\right)-\delta_{h, 2} a\left(u, \phi_{2}\right) \\
\leq & \left(f, u-\hat{u}_{h}\right)+\delta_{h, 1} a\left(u, \phi_{1}\right)-\delta_{h, 2} a\left(u, \phi_{2}\right) \\
= & \left(f, E_{h} \Pi_{h} u-\tilde{u}_{h}\right)+\left(f, u-E_{h} \Pi_{h} u\right) \\
& -\delta_{h, 1}\left[\left(f, \phi_{1}\right)-a\left(u, \phi_{1}\right)\right]+\delta_{h, 2}\left[\left(f, \phi_{2}\right)-a\left(u, \phi_{2}\right)\right],
\end{aligned}
$$

and, we have, because of (2.9),

$$
\left|\left(f, u-E_{h} \Pi_{h} u\right)\right| \leq\|f\|_{L_{2}(\Omega)}\left\|u-E_{h} \Pi_{h} u\right\|_{L_{2}(\Omega)} \leq C h^{3} .
$$

Combining (4.3)-4.11), we find

$$
\begin{aligned}
& a_{h}\left(u, \Pi_{h} u-u_{h}\right)-\left(f, E_{h}\left(\Pi_{h} u-u_{h}\right)\right) \\
& \leq C\left[\left(h+\delta_{h, 1}^{\frac{1}{2}}+\delta_{h, 2}^{\frac{1}{2}}\right)\left\|u_{h}-\Pi_{h} u\right\|_{h}+\left(h^{2}+\delta_{h, 1}+\delta_{h, 2}\right)\right] .
\end{aligned}
$$

The estimate (4.2) follows from (2.7) and (4.12).

Theorem 4.2. There exists a positive constant $C$ independent of $h$ such that

$$
\left\|u-u_{h}\right\|_{h} \leq C h .
$$

Proof. It follows from (2.5), (2.13), Lemma 4.1, and the arithmetic-geometric mean inequality that

$$
\begin{aligned}
\left\|u-u_{h}\right\|_{h}^{2} & \leq C\left[\left\|u-\Pi_{h} u\right\|_{h}^{2}+\left(h+\delta_{h, 1}^{\frac{1}{2}}+\delta_{h, 2}^{\frac{1}{2}}\right)\left\|\Pi_{h} u-u_{h}\right\|_{h}+\left(h^{2}+\delta_{h, 1}+\delta_{h, 2}\right)\right] \\
& \leq C\left[\left(h+\delta_{h, 1}^{\frac{1}{2}}+\delta_{h, 2}^{\frac{1}{2}}\right)\left(\left\|\Pi_{h} u-u\right\|_{h}+\left\|u-u_{h}\right\|_{h}\right)+\left(h^{2}+\delta_{h, 1}+\delta_{h, 2}\right)\right] \\
& \leq C\left(h^{2}+\delta_{h, 1}+\delta_{h, 2}\right)+\frac{1}{2}\left\|u-u_{h}\right\|_{h}^{2}
\end{aligned}
$$


and hence

$$
\left\|u-u_{h}\right\|_{h}^{2} \leq C\left(h^{2}+\delta_{h, 1}+\delta_{h, 2}\right),
$$

which together with (3.29) implies the estimate (4.13).

\section{Concluding Remarks}

The results in this paper are also valid for the one-obstacle problem. In fact, the analysis of the one-obstacle problem is slightly simpler since we only have to deal with one coincidence set.

For simplicity we have assumed $\Omega$ to be convex. But the results can be extended to a nonconvex polygonal domain $\Omega$, in which case $u \in H^{2+\alpha}(\Omega)$ for some $\alpha \in\left(\frac{1}{2}, 1\right)$ (cf. 8, 35, 26, 39]), and the error estimate (4.13) becomes $\left\|u-u_{h}\right\|_{h} \leq C h^{\alpha}$.

It is also possible to extend the results in this paper to obstacle problems with nonhomogeneous boundary conditions. Let $g \in H^{4}(\Omega)$ such that $\psi_{1}<g<\psi_{2}$ on $\partial \Omega$, and

$$
K=\left\{v \in H^{2}(\Omega): v-g \in H_{0}^{2}(\Omega) \quad \text { and } \quad \psi_{1} \leq v \leq \psi_{2} \quad \text { in } \Omega\right\} .
$$

Finite element methods for (1.1)-(1.3) can be developed for this closed convex set $K$. Since there are many different treatments for the nonhomogeneous boundary conditions depending on the choice of the finite element space and the variational form, we prefer to discuss them separately in future work together with numerical solutions of the discrete obstacle problems. Numerical results in 18 for the quadratic $C^{0}$ interior penalty method have confirmed the error estimate (4.13) for obstacle problems with homogeneous or nonhomogeneous boundary conditions.

Finally, we note that the results in [30, 31, 21] also hold for simply supported plates and hence the methodology of this paper can be applied to the obstacle problem for simply supported plates.

\section{Appendix A. $C^{2}$ Regularity for the two-obstacle Problem}

First we will extend the $C^{2}$ regularity result in 31, 21 to the one-obstacle problem with nonhomogeneous data. Let $D \subset \mathbb{R}^{2}$ be a smooth bounded domain (not necessarily connected), $f \in L_{2}(D), \psi \in C^{2}(\bar{D}), \psi<0$ on $\partial D$, and

$$
K_{0, \psi}=\left\{v \in H_{0}^{2}(D): v \geq \psi \text { on } D\right\} .
$$

Consider the following obstacle problem:

Find $z_{0, \psi, f} \in K_{0, \psi}$ such that

$$
z_{0, \psi, f}=\underset{v \in K_{0, \psi}}{\operatorname{argmin}} G_{D}(v),
$$

where the functional $G_{D}$ is defined as in (1.3)-(1.4), but with $\Omega$ replaced by $D$.

Lemma A.1. The solution $z_{0, \psi, f}$ of (A.1) belongs to $C^{2}(\bar{D})$.

Proof. Let $a_{D}(\cdot, \cdot)$ be defined as in (1.4) but with $\Omega$ replaced by $D$, and $\phi \in H_{0}^{2}(D)$ be defined by

$$
a_{D}(\phi, v)=(f, v) \quad \forall v \in H_{0}^{2}(D) .
$$

It follows from elliptic regularity (cf. [3, 42, 37]) and the Sobolev embedding theorem [1 that $\phi \in H^{4}(D) \subset C^{2}(\bar{D})$.

Let $\psi_{*}=\psi-\phi$ and

$$
K_{0, \psi_{*}}=\left\{w \in H_{0}^{2}(D): w \geq \psi_{*} \text { on } D\right\} .
$$


Then we have $\psi_{*} \in C^{2}(\bar{D}), \psi_{*}<0$ on $\partial D$, and $w \in K_{0, \psi_{*}}$ if and only if $v=w+\phi \in$ $K_{0, \psi}$. By a simple calculation using (A.2) we find

$$
\frac{1}{2} a_{D}(w, w)=G_{D}(v)+\frac{1}{2} a_{D}(\phi, \phi),
$$

and hence $z_{*}=z_{0, \psi, f}-\phi \in K_{0, \psi_{*}}$ satisfies

$$
z_{*}=\underset{w \in K_{0, \psi_{*}}}{\operatorname{argmin}} \frac{1}{2} a_{D}(w, w) .
$$

It follows from the $C^{2}$ regularity result in [31, 21] that $z_{*} \in C^{2}(\bar{D})$ and hence $z_{0, \psi, f}=z_{*}+\phi \in C^{2}(\bar{D})$.

Next we consider the obstacle problem of finding $z_{g, \psi, f} \in K_{g, \psi}$ such that

$$
z_{g, \psi, f}=\underset{v \in K_{g, \psi}}{\operatorname{argmin}} G_{D}(v),
$$

where $g \in H^{4}(D) \subset C^{2}(\bar{D})$ satisfies $g>\psi$ on $\partial D$ and

$$
K_{g, \psi}=\left\{v \in H^{2}(D): v-g \in H_{0}^{2}(D) \text { and } v \geq \psi \text { on } D\right\} .
$$

Lemma A.2. The solution $z_{g, \psi, f}$ of (A.3) belongs to $C^{2}(\bar{D})$.

Proof. Let $\psi_{\dagger}=\psi-g$ and

$$
K_{0, \psi_{\dagger}}=\left\{w \in H_{0}^{2}(D): w \geq \psi_{\dagger}\right\} .
$$

Then we have $\psi_{\dagger} \in C^{2}(\bar{D}), \psi_{\dagger}<0$ on $\partial D$, and $w \in K_{0, \psi_{\dagger}}$ if and only if $v=w+g \in$ $K_{g, \psi}$. By another simple calculation we find

$$
\frac{1}{2} a_{D}(w, w)-\left(f-\Delta^{2} g, w\right)=G_{D}(v)-\frac{1}{2} a_{D}(g, g)+(f, g)
$$

and hence $z_{\dagger}=z_{g, \psi, f}-g \in K_{0, \psi_{\dagger}}$ satisfies

$$
z_{\dagger}=\underset{w \in K_{0, \psi_{\dagger}}}{\operatorname{argmin}}\left[\frac{1}{2} a_{D}(w, w)-\left(f-\Delta^{2} g, w\right)\right],
$$

i.e., $z_{\dagger}=z_{0, \psi_{\dagger},\left(f-\Delta^{2} g\right)}$ in the notation of A.1 . Therefore Lemma A.1 implies that $z_{\dagger} \in C^{2}(\bar{D})$ and hence $z_{g, \psi, f}=z_{\dagger}+g \in C^{2}(\bar{D})$.

We are now ready to tackle the two-obstacle problem defined by (1.1)-(1.3). Since (1.5) implies $\Delta^{2} u=f$ on $\Omega \backslash\left(I_{1} \cup I_{2}\right)$, from interior elliptic regularity (cf. [3, 42, 37]) we know that $u \in H_{\text {loc }}^{4}\left(\Omega \backslash\left(I_{1} \cup I_{2}\right)\right)$ and hence $u$ is $C^{2}$ outside the coincidence sets $I_{1}$ and $I_{2}$.

Let $D \subset \Omega$ be a smooth domain such that $I_{1} \subset D$ and $\bar{D}$ is disjoint from $\partial \Omega$ and $I_{2}$. Since $u$ belongs to the Sobolev space $H^{4}(\mathcal{N})$ in an open neighborhood $\mathcal{N}$ of $\partial D$, we have $u=g$ on $\partial D$, where $g \in H^{4}(D)$ is the product of $u$ and a smooth cut-off function. Because $\partial D$ is outside the coincidence set $I_{1}$, we also have $g>\psi_{1}$ on $\partial D$.

We claim that $u_{D}=\left.u\right|_{D}$ satisfies

$$
u_{D}=\underset{v \in K_{*}}{\operatorname{argmin}} G_{D}(v),
$$

where

$$
K_{*}=\left\{v \in H^{2}(D): v-g \in H_{0}^{2}(D) \text { and } \psi_{1} \leq v \leq \psi_{2} \text { on } D\right\} .
$$


Indeed, if there exists a function $v_{*} \in K_{*}$ such that $G_{D}\left(v_{*}\right)<G_{D}\left(u_{D}\right)$, then the function $u_{*}$ defined by

$$
u_{*}(x)= \begin{cases}v_{*}(x) & \text { if } x \in D \\ u(x) & \text { if } x \in \Omega \backslash D\end{cases}
$$

belongs to $K$ and $G\left(u_{*}\right)<G(u)$, which is impossible because of (1.1).

Next we claim that $u_{D}$ also satisfies

$$
u_{D}=\underset{v \in K_{g, \psi_{1}}}{\operatorname{argmin}} G_{D}(v),
$$

where

$$
K_{g, \psi_{1}}=\left\{v \in H^{2}(D): v-g \in H_{0}^{2}(D) \text { and } v \geq \psi_{1} \text { on } D\right\} .
$$

We will establish (A.5) by showing that $u_{D}$ satisfies the equivalent variational inequality

$$
a_{D}\left(u_{D}, v-u_{D}\right) \geq\left(f, v-u_{D}\right) \quad \forall v \in K_{g, \psi_{1}} .
$$

Let $v \in K_{g, \psi_{1}}$ be arbitrary. Since $u_{D}$ is strictly less than $\psi_{2}$ on the compact set $\bar{D}$, the function $w_{t}=(1-t) u_{D}+t v$ belongs to $K_{*}$ for any sufficiently small nonnegative number $t$ and $G_{D}\left(u_{D}\right) \leq G_{D}\left(w_{t}\right)$ for such $t$ because of (A.4). It follows that $(d / d t) G_{D}\left(w_{t}\right)$ is non-negative at $t=0$, which is equivalent to (A.6).

In view of (A.5) and Lemma A.2, we have $u_{D}=z_{g, \psi_{1}, f} \in C^{2}(\bar{D})$. Similarly, we can prove that $u$ is $C^{2}$ in a neighborhood of the coincidence set $I_{2}$. We conclude that $u \in C^{2}(\Omega)$.

\section{Appendix B. Estimate (2.8) For the Morley finite element space}

A function $v$ belongs to the Morley finite element space $V_{h}$ if and only if (i) $\left.v\right|_{T} \in P_{2}(T)$ for all $T \in \mathcal{T}_{h}$, (ii) $v$ is continuous at the vertices of $\mathcal{T}_{h}$ and vanishes at the vertices along $\partial \Omega$, and (iii) the normal derivative of $v$ is continuous at the midpoints of the edges in $\mathcal{T}_{h}$ and vanishes at the midpoints of the edges along $\partial \Omega$. Note that $\nabla v$ (and hence $\nabla\left(v-E_{h} v\right)$ ) is continuous at the midpoints of the edges in $\mathcal{E}_{h}$ and vanishes at the midpoints of the edges along $\partial \Omega$.

Let $\zeta \in H^{3}(\Omega) \cap H_{0}^{2}(\Omega), v \in V_{h}$ and $v_{T}$ be the restriction of $v$ to a triangle $T \in \mathcal{T}_{h}$. Using integration by parts and the midpoint rule, we find

$$
\begin{aligned}
& a_{h}\left(\zeta, v-E_{h} v\right)=\sum_{T \in \mathcal{T}_{h}} \int_{T} D^{2} \zeta: D^{2}\left(v-E_{h} v\right) d x \\
& =-\sum_{T \in \mathcal{T}_{h}} \int_{T} \Delta(\nabla \zeta) \cdot \nabla\left(v-E_{h} v\right) d x+\sum_{T \in \mathcal{T}_{h}} \int_{\partial T} D^{2} \zeta:\left[\nabla\left(v_{T}-E_{h} v\right) \otimes \boldsymbol{n}_{T}\right] d s \\
& =-\sum_{T \in \mathcal{T}_{h}} \int_{T} \Delta(\nabla \zeta) \cdot \nabla\left(v-E_{h} v\right) d x \\
& \left.\quad+\sum_{e \in \mathcal{E}_{h}} \int_{e}\left[D^{2} \zeta-\overline{\left(D^{2} \zeta\right)_{e}}\right]:\left[\nabla \nabla\left(v-E_{h} v\right) \otimes \boldsymbol{n}\right]\right]_{e} d s,
\end{aligned}
$$

where $\boldsymbol{n}_{T}$ is the unit outward normal along $\partial T, \overline{\left(D^{2} \zeta\right)_{e}}$ is the mean of $D^{2} \zeta$ over the edge $e$ and $\left[\left[\nabla\left(v-E_{h} v\right) \times \boldsymbol{n}\right]\right]_{e}$ is the sum of $\nabla\left(v_{T}-E_{h} v\right) \otimes \boldsymbol{n}_{T}$ over the triangles that share $e$ as a common edge. 
It follows that

$$
\begin{aligned}
& \left|a_{h}\left(\zeta, v-E_{h} v\right)\right| \leq|\Delta \zeta|_{H^{1}(\Omega)}\left(\sum_{T \in \mathcal{T}_{h}}\left|v-E_{h} v\right|_{H^{1}(T)}^{2}\right)^{1 / 2} \\
& \quad+\left(\sum_{e \in \mathcal{E}_{h}}|e|^{-1}\left\|D^{2} \zeta-\overline{\left(D^{2} \zeta\right)_{e}}\right\|_{L_{2}(e)}^{2}\right)^{1 / 2}\left(\sum_{e \in \mathcal{E}_{h}}|e|\left\|\left[\left[\nabla\left(v-E_{h} v\right) \otimes \boldsymbol{n}\right]\right]\right\|_{L_{2}(e)}^{2}\right)^{1 / 2} \\
& \quad \leq C|\zeta|_{H^{3}(\Omega)}\left(\sum_{T \in \mathcal{T}_{h}}\left|v-E_{h} v\right|_{H^{1}(T)}^{2}\right)^{1 / 2} \leq C h|\zeta|_{H^{3}(\Omega)}\|v\|_{h},
\end{aligned}
$$

where we have used the Cauchy-Schwarz inequality, the trace theorem (with scaling), the Bramble-Hilbert lemma [10] and (2.7).

\section{Appendix C. Finite element methods for the Biharmonic Problem}

When the obstacles are absent we have $K=H_{0}^{2}(\Omega), K_{h}=V_{h}$, and the finite element methods in Section 2 become finite element methods for the biharmonic problem. A concise unified analysis of these methods can be performed using the tools introduced in Section 2 ,

Let $z \in H_{0}^{2}(\Omega)$ and $z_{h} \in V_{h}$ satisfy

$$
\begin{aligned}
a(z, v) & =(f, v) & \forall v \in H_{0}^{2}(\Omega), \\
a_{h}\left(z_{h}, v\right) & =(f, v) & \forall v \in V_{h},
\end{aligned}
$$

where $f \in L_{2}(\Omega)$. The convexity of $\Omega$ implies that $z \in H^{3}(\Omega)$ and

$$
\|z\|_{H^{3}(\Omega)} \leq C_{\Omega}\|f\|_{L_{2}(\Omega)} .
$$

We have a standard abstract error estimate [16, Lemma 10.1.1]

$$
\left\|z-z_{h}\right\|_{h} \leq C\left(\inf _{v \in V_{h}}\|z-v\|_{h}+\sup _{w \in V_{h} \backslash\{0\}}\left[a_{h}(z, w)-(f, w)\right] /\|w\|_{h}\right)
$$

that follows from (2.2), (2.3) and (C.2), and for any $w \in V_{h}$, we have

$$
\begin{aligned}
a_{h}(z, w)-(f, w) & =a_{h}\left(z, w-E_{h} w\right)-\left(f, w-E_{h} w\right) \\
& \leq C\left[h|z|_{H^{3}(\Omega)}+h^{2}\|f\|_{L_{2}(\Omega)}\right]\|w\|_{h}
\end{aligned}
$$

by (2.1), (2.7), (2.8) and (C.1).

Combining (2.5) and (C.3)

$$
\left\|z-z_{h}\right\|_{h} \leq C h\|f\|_{L_{2}(\Omega)} .
$$

\section{REFERENCES}

[1] R.A. Adams and J.J.F. Fournier. Sobolev Spaces (Second Edition). Academic Press, Amsterdam, 2003. MR2424078 (2009e:46025)

[2] A. Adini and R.W. Clough. Analysis of plate bending by the finite element method. Technical Report G. 7337, NSF, 1961.

[3] S. Agmon. Lectures on Elliptic Boundary Value Problems. Van Nostrand, Princeton, 1965. MR0178246 (31:2504)

[4] R. An. Discontinuous Galerkin finite element method for the fourth-order obstacle problem. Appl. Math. Comput., 209:351-355, 2009. MR2493411

[5] J.H. Argyris, I. Fried, and D.W. Scharpf. The TUBA family of plate elements for the matrix displacement method. Aero. J. Roy. Aero. Soc., 72:701-709, 1968.

[6] J.P. Aubin. Approximation of variational inequations. In Functional Analysis and Optimization, pages 7-14. Academic Press, New York, 1966. MR0213889 (35:4743) 
[7] G.P. Bazeley, Y.K. Cheung, B.M. Irons, and O.C. Zienkiewicz. Triangular elements in bending - conforming and nonconforming solutions. In Proceedings of the Conference on Matrix Methods in Structural Mechanics. Wright Patterson A.F.B., Ohio, 1965.

[8] H. Blum and R. Rannacher. On the boundary value problem of the biharmonic operator on domains with angular corners. Math. Methods Appl. Sci., 2:556-581, 1980. MR595625 (82a:35022)

[9] F.K. Bogner, R.L. Fox, and L.A. Schmit. The generation of interelement compatible stiffness and mass matrices by the use of interpolation formulas. In Proceedings Conference on Matrix Methods in Structural Mechanics, pages 397-444. Wright Patterson A.F.B., Dayton, OH, 1965.

[10] J.H. Bramble and S.R. Hilbert. Estimation of linear functionals on Sobolev spaces with applications to Fourier transforms and spline interpolation. SIAM J. Numer. Anal., 7:113-124, 1970. MR0263214(41:7819)

[11] S.C. Brenner. Two-level additive Schwarz preconditioners for nonconforming finite elements. In D.E. Keyes and J. Xu, editors, Domain Decomposition Methods in Scientific and Engineering Computing, pages 9-14. Amer. Math. Soc., Providence, 1994. Contemporary Mathematics 180. MR 1312372 (95j:65134)

[12] S.C. Brenner. A two-level additive Schwarz preconditioner for nonconforming plate elements. Numer. Math., 72:419-447, 1996. MR1376107 (97h:65147)

[13] S.C. Brenner. Two-level additive Schwarz preconditioners for nonconforming finite element methods. Math. Comp., 65:897-921, 1996. MR1348039(96j:65117)

[14] S.C. Brenner. Convergence of nonconforming multigrid methods without full elliptic regularity. Math. Comp., 68:25-53, 1999. MR.1620215 (99c:65229)

[15] S.C. Brenner, T. Gudi, and L.-Y. Sung. An a posteriori error estimator for a quadratic $C^{0}$ interior penalty method for the biharmonic problem. IMA J. Numer. Anal., 30:777-798, 2010. MR2670114

[16] S.C. Brenner and L.R. Scott. The Mathematical Theory of Finite Element Methods (Third Edition). Springer-Verlag, New York, 2008. MR2373954(2008m:65001)

[17] S.C. Brenner and L.-Y. Sung. $C^{0}$ interior penalty methods for fourth order elliptic boundary value problems on polygonal domains. J. Sci. Comput., 22/23:83-118, 2005. MR2142191 (2005m:65258)

[18] S.C. Brenner, L.-Y. Sung, H. Zhang, and Y. Zhang. A quadratic $C^{0}$ interior penalty method for the displacement obstacle problem of clamped Kirchhoff plates. preprint, 2011.

[19] H. Brezis. Analyse Fonctionnelle. Masson, Paris, 1983. MR697382 (85a:46001)

[20] F. Brezzi, W. Hager, and P.-A. Raviart. Error estimates for the finite element solution of variational inequalities. Numer. Math., 28:431-443, 1977. MR0448949 (56:7254)

[21] L.A. Caffarelli and A. Friedman. The obstacle problem for the biharmonic operator. Ann. Scuola Norm. Sup. Pisa Cl. Sci. (4), 6:151-184, 1979. MR529478 (80g:35049)

[22] L.A. Caffarelli, A. Friedman, and A. Torelli. The two-obstacle problem for the biharmonic operator. Pacific J. Math., 103:325-335, 1982. MR705233 (85g:35056)

[23] P.G. Ciarlet. Sur l'élément de Clough et Tocher. RAIRO Anal. Numér., 8:19-27, 1974. MR0381349(52:2246)

[24] P.G. Ciarlet. The Finite Element Method for Elliptic Problems. North-Holland, Amsterdam, 1978. MR0520174 (58:25001)

[25] $\mathrm{Ph}$. Clément. Approximation by finite element functions using local regularization. RAIRO Anal. Numér., 9:77-84, 1975. MR0400739 (53:4569)

[26] M. Dauge. Elliptic Boundary Value Problems on Corner Domains, Lecture Notes in Mathematics 1341. Springer-Verlag, Berlin-Heidelberg, 1988. MR.961439 (91a:35078)

[27] B.F. de Veubeke. Variational principles and the patch test. Internat. J. Numer. Methods Engrg., 8:783-801, 1974. MR0375911 (51:12099)

[28] G. Engel, K. Garikipati, T.J.R. Hughes, M.G. Larson, L. Mazzei, and R.L. Taylor. Continuous/discontinuous finite element approximations of fourth order elliptic problems in structural and continuum mechanics with applications to thin beams and plates, and strain gradient elasticity. Comput. Methods Appl. Mech. Engrg., 191:3669-3750, 2002. MR1915664 (2003d:74086)

[29] R.S. Falk. Error estimates for the approximation of a class of variational inequalities. Math. Comp., 28:963-971, 1974. MR0391502(52:12323) 
[30] J. Frehse. Zum Differenzierbarkeitsproblem bei Variationsungleichungen höherer Ordnung. Abh. Math. Sem. Univ. Hamburg, 36:140-149, 1971. MR0330754 (48:9091)

[31] J. Frehse. On the regularity of the solution of the biharmonic variational inequality. Manuscripta Math., 9:91-103, 1973. MR0324208 (48:2560)

[32] A. Friedman. Variational Principles and Free-Boundary Problems. Robert E. Krieger Publishing Co. Inc., Malabar, FL, second edition, 1988. MR.1009785 (90k:35271)

[33] V. Girault and L.R. Scott. Hermite interpolation of nonsmooth functions preserving boundary conditions. Math. Comp., 71:1043-1074 (electronic), 2002. MR.1898745 (2003e:65012)

[34] R. Glowinski, J.-L. Lions, and R. Trémolières. Numerical Analysis of Variational Inequalities. North-Holland Publishing Co., Amsterdam, 1981. MR635927 (83k:49014)

[35] P. Grisvard. Elliptic Problems in Non Smooth Domains. Pitman, Boston, 1985.

[36] I. Hlaváček, J. Haslinger, J. Nečas, and J. Lovíšek. Solution of Variational Inequalities in Mechanics. Springer-Verlag, New York, 1988. MR952855 (89i:73003)

[37] L. Hörmander. Linear Partial Differential Operators. Springer-Verlag, Berlin, 1976. MR0404822(53:8622)

[38] D. Kinderlehrer and G. Stampacchia. An Introduction to Variational Inequalities and Their Applications. SIAM, Philadelphia, 2000. MR.1786735(2002d:49001)

[39] V.A. Kozlov, V.G. Maz'ya, and J. Rossmann. Spectral Problems Associated with Corner Singularities of Solutions to Elliptic Equations. AMS, Providence, 2001. MR.178891 (2001i:35069)

[40] P.D. Lax. Functional Analysis. Wiley-Interscience, New York, 2002. MR.1892228 (2003a:47001)

[41] J.-L. Lions and G. Stampacchia. Variational inequalities. Comm. Pure Appl. Math., 20:493519, 1967. MR0216344(35:7178)

[42] J.L. Lions and E. Magenes. Non-homogeneous Boundary Value Problems and Applications I. Springer-Verlag, New York, 1972. MR0350177 (50:2670)

[43] L.S.D. Morley. The triangular equilibrium problem in the solution of plate bending problems. Aero. Quart., 19:149-169, 1968.

[44] J. Nečas. Les Méthodes Directes en Théorie des Équations Elliptiques. Masson, Paris, 1967. MR.0227584 (37:3168)

[45] J.-F. Rodrigues. Obstacle Problems in Mathematical Physics. North-Holland Publishing Co., Amsterdam, 1987. MR880369 (88d:35006)

[46] B. Schild. A regularity result for polyharmonic variational inequalities with thin obstacles. Ann. Scuola Norm. Sup. Pisa Cl. Sci. (4), 11:87-122, 1984. MR752581 (85k:35101)

[47] L.R. Scott and S. Zhang. Finite element interpolation of nonsmooth functions satisfying boundary conditions. Math. Comp., 54:483-493, 1990. MR 1011446 (90j:65021)

[48] Z. Shi. On the convergence of the incomplete biquadratic nonconforming plate element. Math. Numer. Sinica, 8:53-62, 1986. MR:864031 (87j:73084)

[49] L.-H. Wang. Error estimates of two nonconforming finite elements for the obstacle problem. J. Comput. Math., 4:11-20, 1986. MR854380 (87k:65149)

[50] L.-H. Wang. Some nonconforming finite element approximations for a fourth order variational inequality with displacement obstacle. Chinese J. Numer. Math. Appl., 13:1-6, 1991. MR 1136145 (92h:65175)

[51] L.-H. Wang. On the quadratic finite element approximation to the obstacle problem. Numer. Math., 92:771-778, 2002. MR.1935809(2003i:65118)

[52] A. Ženíšek. Interpolation polynomials on the triangle. Numer. Math., 15:283-296, 1970. MR0275014(43:772)

Department of Mathematics and Center for Computation and Technology, Louisiana

State University, Baton Rouge, Louisiana 70803

E-mail address: brenner@math.1su.edu

Department of Mathematics and Center for Computation and Technology, Louisiana

State University, Baton Rouge, Louisiana 70803

E-mail address: sung@math.lsu.edu

Department of Mathematics and Center for Computation and Technology, Louisiana

State University, Baton Rouge, Louisiana 70803

E-mail address: yzhang24@math.1su.edu 